\title{
Proton-proton interactions and onset of deconfinement
}

A. Aduszkiewicz, ${ }^{15}$ E. V. Andronov, ${ }^{21}$ T. Antićić, ${ }^{3}$ V. Babkin, ${ }^{19}$ M. Baszczyk,,${ }^{13}$ S. Bhosale, ${ }^{10}$ A. Blondel,,${ }^{23}$ M. Bogomilov, ${ }^{2}$ A. Brandin, ${ }^{20}$ A. Bravar, ${ }^{23} \mathrm{~W}$. Bryliński, ${ }^{17} \mathrm{~J}$. Brzychczyk, ${ }^{12} \mathrm{M}$. Buryakov, ${ }^{19} \mathrm{O}$. Busygina, ${ }^{18} \mathrm{~A}$. Bzdak, ${ }^{13} \mathrm{H}$. Cherif, ${ }^{6}$

M. Ćirković, ${ }^{22}$ M. Csanad, ${ }^{7}$ J. Cybowska, ${ }^{17}$ T. Czopowicz, $,{ }^{9}, 17$ A. Damyanova,${ }^{23}$ N. Davis, ${ }^{10}$ M. Deliyergiyev, ${ }^{9}$ M. Deveaux ${ }^{6}$ A. Dmitriev, ${ }^{19}$ W. Dominik, ${ }^{15}$ P. Dorosz,${ }^{13}$ J. Dumarchez,${ }^{4}$ R. Engel,${ }^{5}$ G. A. Feofilov, ${ }^{21}$ L. Fields,${ }^{24}$ Z. Fodor,${ }^{7,16}$ A. Garibov, ${ }^{1}$ M. Gaździcki, ${ }^{6,9}$ O. Golosov, ${ }^{20}$ V. Golovatyuk, ${ }^{19}$ M. Golubeva, ${ }^{18}$ K. Grebieszkow, ${ }^{17}$ F. Guber, ${ }^{18}$ A. Haesler, ${ }^{23}$ S. N. Igolkin, ${ }^{21}$ S. Ilieva, ${ }^{2}$ A. Ivashkin, ${ }^{18}$ S. R. Johnson, ${ }^{25}$ K. Kadija, ${ }^{3}$ E. Kaptur, ${ }^{14}$ N. Kargin, ${ }^{20}$ E. Kashirin, ${ }^{20}$ M. Kiełbowicz, ${ }^{10}$

V. A. Kireyeu, ${ }^{19}$ V. Klochkov, ${ }^{6}$ V. I. Kolesnikov, ${ }^{19}$ D. Kolev, ${ }^{2}$ A. Korzenev, ${ }^{23}$ V. N. Kovalenko, ${ }^{21}$ S. Kowalski, ${ }^{14}$ M. Koziel, ${ }^{6}$ A. Krasnoperov, ${ }^{19}$ W. Kucewicz, ${ }^{13}$ M. Kuich, ${ }^{15}$ A. Kurepin, ${ }^{18}$ D. Larsen, ${ }^{12}$ A. László, ${ }^{7}$ T. V. Lazareva, ${ }^{21}$ M. Lewicki, ${ }^{16}$

K. Łojek, ${ }^{12}$ B. Łysakowski, ${ }^{14}$ V. V. Lyubushkin, ${ }^{19}$ M. Maćkowiak-Pawłowska,,${ }^{17}$ Z. Majka, ${ }^{12}$ B. Maksiak, ${ }^{11}$ A. I. Malakhov, ${ }^{19}$ A. Marcinek, ${ }^{10}$ A. D. Marino, ${ }^{25}$ K. Marton,${ }^{7}$ H.-J. Mathes, ${ }^{5}$ T. Matulewicz, ${ }^{15}$ V. Matveev, ${ }^{19}$ G. L. Melkumov, ${ }^{19}$

A. O. Merzlaya, ${ }^{12}$ B. Messerly, ${ }^{26}$ Ł. Mik, ${ }^{13}$ S. Morozov, ${ }^{18,20}$ S. Mrówczyński, ${ }^{9}$ Y. Nagai, ${ }^{25}$ M. Naskręt, ${ }^{16}$ V. Ozvenchuk, ${ }^{10}$ V. Paolone, ${ }^{26}$ O. Petukhov, ${ }^{18}$ R. Płaneta, ${ }^{12}$ P. Podlaski, ${ }^{15}$ B. A. Popov,${ }^{19,4}$ B. Porfy,${ }^{7}$ M. Posiadała-Zezula, ${ }^{15}$

D. S. Prokhorova, ${ }^{21}$ D. Pszczel, ${ }^{11}$ S. Puławski $\odot,{ }^{14}$ J. Puzović, ${ }^{22}$ M. Ravonel,${ }^{23}$ R. Renfordt, ${ }^{6}$ E. Richter-Wąs, ${ }^{12}$ D. Röhrich, ${ }^{8}$ E. Rondio, ${ }^{11}$ M. Roth, ${ }^{5}$ B. T. Rumberger, ${ }^{25}$ M. Rumyantsev,${ }^{19}$ A. Rustamov,${ }^{1,6}$ M. Rybczynski, ${ }^{9}$ A. Rybicki,${ }^{10}$ A. Sadovsky,${ }^{18}$ K. Schmidt, ${ }^{14}$ I. Selyuzhenkov, ${ }^{20}$ A. Yu. Seryakov, ${ }^{21}$ P. Seyboth, ${ }^{9}$ M. Stodkowski,,${ }^{17}$ P. Staszel, ${ }^{12}$ G. Stefanek, ${ }^{9}$ J. Stepaniak, ${ }^{11}$ M. Strikhanov, ${ }^{20}$ H. Ströbele, ${ }^{6}$ T. Šuša, ${ }^{3}$ A. Taranenko,${ }^{20}$ A. Tefelska, ${ }^{17}$ D. Tefelski, ${ }^{17}$ V. Tereshchenko, ${ }^{19}$ A. Toia, ${ }^{6}$ R. Tsenov, ${ }^{2}$ L. Turko, ${ }^{16}$ R. Ulrich, ${ }^{5}$ M. Unger, ${ }^{5}$ F. F. Valiev, ${ }^{21}$ D. Veberič,${ }^{5}$ V. V. Vechernin,,${ }^{21}$ A. Wickremasinghe, ${ }^{26}$ Z. Włodarczyk, ${ }^{9}$ O. Wyszyński, ${ }^{12}$ E. D. Zimmerman, ${ }^{25}$ and R. Zwaska ${ }^{24}$

(NA61/SHINE Collaboration)

${ }^{1}$ National Nuclear Research Center, Baku, Azerbaijan

${ }^{2}$ Faculty of Physics, University of Sofia, Sofia, Bulgaria

${ }^{3}$ Ruđer Bošković Institute, Zagreb, Croatia

${ }^{4}$ LPNHE, University of Paris VI and VII, Paris, France

${ }^{5}$ Karlsruhe Institute of Technology, Karlsruhe, Germany

${ }^{6}$ University of Frankfurt, Frankfurt, Germany

${ }^{7}$ Wigner Research Centre for Physics of the Hungarian Academy of Sciences, Budapest, Hungary

${ }^{8}$ University of Bergen, Bergen, Norway

${ }^{9}$ Jan Kochanowski University, Kielce, Poland

${ }^{10}$ Institute of Nuclear Physics, Polish Academy of Sciences, Kraków, Poland

${ }^{11}$ National Centre for Nuclear Research, Warsaw, Poland

${ }^{12}$ Jagiellonian University, Kraków, Poland

${ }^{13}$ AGH-University of Science and Technology, Kraków, Poland

${ }^{14}$ University of Silesia, Katowice, Poland

${ }^{15}$ University of Warsaw, Warsaw, Poland

${ }^{16}$ University of Wroctaw, Wroctaw, Poland

${ }^{17}$ Warsaw University of Technology, Warsaw, Poland

${ }^{18}$ Institute for Nuclear Research, Moscow, Russia

${ }^{19}$ Joint Institute for Nuclear Research, Dubna, Russia

${ }^{20}$ National Research Nuclear University (Moscow Engineering Physics Institute), Moscow, Russia

${ }^{21}$ St. Petersburg State University, St. Petersburg, Russia

${ }^{22}$ University of Belgrade, Belgrade, Serbia

${ }^{23}$ University of Geneva, Geneva, Switzerland

${ }^{24}$ Fermilab, Batavia, Illinois 60510, USA

${ }^{25}$ University of Colorado, Boulder, Colorado 80309, USA

${ }^{26}$ University of Pittsburgh, Pittsburgh, Pennsylvania 15260, USA

(Received 23 December 2019; accepted 9 June 2020; published 15 July 2020)

Published by the American Physical Society under the terms of the Creative Commons Attribution 4.0 International license. Further distribution of this work must maintain attribution to the author(s) and the published article's title, journal citation, and DOI. Funded by $S C O A P^{3}$. 
The experiment of the NA61/SHINE Collaboration at the CERN SPS is performing a unique study of the phase diagram of strongly interacting matter by varying collision energy and nuclear mass number of colliding nuclei. In central $\mathrm{Pb}+\mathrm{Pb}$ collisions, the experiment of the NA49 Collaboration found structures in the energy dependence of several observables in the energy range of the CERN SPS that had been predicted for the transition to a deconfined phase. New measurements of the NA61/SHINE Collaboration find intriguing similarities in $p+p$ interactions for which no deconfinement transition is expected at the energies of the SPS. Possible implications will be discussed.

DOI: 10.1103/PhysRevC.102.011901

The standard approach to heavy-ion collisions [1] assumes creation of strongly interacting matter in local equilibrium at the early stage of a collision. The matter properties depend on energy and baryon densities via an equation of state. The matter expansion is modeled by hydrodynamics and its conversion to final-state hadrons by statistical hadronization models [2-4]. The early stage energy density monotonically increases with collision energy, and, at sufficiently high energies, the state of matter is expected to change from the confined phase to the quark-gluon plasma (QGP).

In an energy scan of central $\mathrm{Pb}+\mathrm{Pb}$ collisions at the CERN SPS, the experiment of the NA49 Collaboration found structures in a common narrow energy interval $\sqrt{s_{N N}} \approx$ 7-12 GeV [5-7] $\left(\sqrt{s_{N N}}\right.$ is the collision energy per nucleon pair in the center-of-mass system) for several observables that had been predicted [8] for the transition to the QGP phase. The most conclusive are as follows:

(i) a fast rise and and sharp peak in the ratio of strangeness to entropy production,

(ii) a fast rise and following plateau of the temperature as measured by the inverse slope parameter of the kaon transverse mass distributions.

Experimental results on proton-proton $(p+p)$ interactions served as an important reference with respect to which new physics in heavy-ion collisions was searched for. The most popular models of $p+p$ interactions are qualitatively different from the standard approach to heavy-ion collisions. They are resonance-string models [9] in which the hydrodynamic expansion of the strongly interacting matter created in nucleus-nucleus $(A+A)$ collisions is replaced in $p+p$ collisions by excitation of resonances or strong fields between color charges of quarks and diquarks (strings). The assumption of statistical hadronization of matter is substituted by dynamical modeling of resonance and/or string decays as well as quark/gluon fragmentation into hadrons. Since the early days, the different modeling of $p+p$ interactions and heavy-ion collisions was supported by qualitative disagreement of the $p+p$ data with predictions of statistical and hydrodynamical models-large particle multiplicity fluctuations and a power-law shape of transverse momentum spectra at high $p_{\mathrm{T}}$ [10]. On the other hand, the different modeling has been questioned by striking agreement of the $p+p$ data with other predictions of statistical and hydrodynamical models - mean multiplicities of hadrons and transverse mass spectra at low and intermediate $p_{\mathrm{T}}$ 's follow a similar pattern. Moreover, recent data of the Large Hadron Collider (LHC) on the azimuthal angle distribution of charged particles in high multiplicity $p+p$ interactions [11-13] show anisotropies up to now observed only in heavy-ion collisions and attributed to the hydrodynamical expansion of matter [14]. Also, it was reported that relative strange particle yields in $p+p$ interactions at the LHC smoothly increase with increasing charged particle multiplicity and, for high multiplicity collisions, are close to those in $\mathrm{Pb}+\mathrm{Pb}$ collisions [15]. This suggests that strongly interacting matter may also be produced in $p+p$ interactions at energies of the LHC with sufficiently high particle multiplicity.

This Rapid Communication addresses the relation between the observation of effects possibly indicating the onset of deconfinement in $\mathrm{Pb}+\mathrm{Pb}$ collisions and the recently uncovered, still unexplained, features in $p+p$ interactions.

New experimental insight is possible thanks to recent results on $p+p$ interactions at the CERN SPS from the fixed target large acceptance hadron detector of the NA61/SHINE Collaboration [16]. The measurements $[17,18]$ cover the energy range in which experimental effects attributed to the onset of deconfinement in heavy-ion collisions are located. They allow to significantly extend and improve the world data on the $\mathrm{K}^{+} / \pi^{+}$ratio $[19,20]$ and the inverse slope parameter $T$ of transverse mass spectra of kaons [21]. Furthermore, recent data on $p+p$ interactions at energies of the LHC allow to establish the collision energy dependence of bulk hadron production properties in the energy range in which the quark-gluon plasma is likely to be created in heavy-ion collisions.

The energy dependence of the $\mathrm{K}^{+} / \pi^{+}$ratio at midrapidity and in the full phase space for inelastic $p+p$ interactions is shown in Figs. 1(a) and 1(b), respectively. The results for heavy-ion $(\mathrm{Pb}+\mathrm{Pb}$ and $\mathrm{Au}+\mathrm{Au})$ collisions are plotted for comparison. The results on $p+p$ interactions of the NA61/SHINE Collaboration at energies of the CERN SPS are shown together with the world data [19,20,22-39]. Results on the midrapidity ratio (the top-left plot) cover the range from low energy of the SPS to the energies of the LHC. The $p+p$ data on the full phase-space ratio (the top-right plot) extends only to $\sqrt{s_{N N}} \approx 50 \mathrm{GeV}$, whereas the heavy-ion data reach $200 \mathrm{GeV}$. The energy dependence of the midrapidity and full phase-space ratio in inelastic $p+p$ interactions is similar. This seems to be also true for heavy-ion collisions. The collision energy dependence of the $\mathrm{K}^{+} / \pi^{+}$ratio in heavyion collisions shows the so-called horn structure. Following a fast rise, the ratio passes through a maximum in the range of the SPS and then settles to a plateau value at higher energies. 

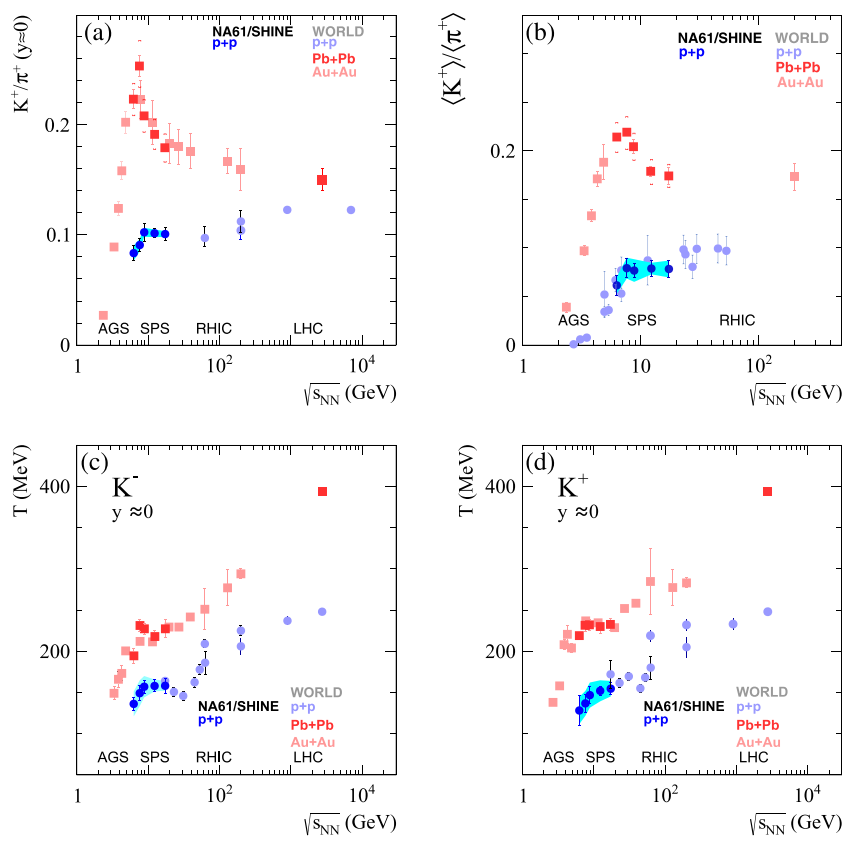

FIG. 1. Energy dependence of the $\mathrm{K}^{+} / \pi^{+}$ratio (a) at midrapidity and (b) in the full phase space as well as (c) the inverse slope parameter $T$ of transverse mass spectra at midrapidity for $\mathrm{K}^{-}$and (d) $\mathrm{K}^{+}$mesons. The results for inelastic $p+p$ interactions of the NA61/SHINE Collaboration (blue circles) are shown together with the world data on $p+p$ interactions (light blue circles) as well as central $\mathrm{Pb}+\mathrm{Pb}$ (red squares) and $\mathrm{Au}+\mathrm{Au}$ (light red squares) collisions [21,23,25-39]. The points of the NA61/SHINE Collaboration include statistical uncertainties, and the systematic uncertainties are marked as a shaded band. For the $\mathrm{K}^{+} / \pi^{+}$and $\left\langle\mathrm{K}^{+}\right\rangle /\left\langle\pi^{+}\right\rangle$ratios, the total uncertainties are marked for the points of the $(\mathrm{Pb}+\mathrm{Pb}$ collisions) of the NA49 Collaboration, the $(p+p, \mathrm{Au}+\mathrm{Au}$ collisions $)$ of the STAR Collaboration, of the PHENIX Collaboration, the $(\mathrm{Au}+\mathrm{Au}$ collisions) of the BRAHMS Collaboration, and the $(p+p$, $\mathrm{Pb}+\mathrm{Pb}$ collisions) experiments of the ALICE Collaboration, whereas only statistical uncertainties are plotted for the remaining points. For the inverse slope parameter $T$ of transverse mass spectra, the total uncertainties are marked for the points of the $(\mathrm{Pb}+\mathrm{Pb}$ collisions) of the NA49 Collaboration, whereas only statistical uncertainties are plotted for the remaining points.

The $\mathrm{K}^{+} / \pi^{+}$ratio at energies of the SPS was shown to be a good measure of the strangeness to entropy ratio [7] which is different in the confined phase (hadrons) and the QGP (quarks, antiquarks, and gluons). This is because, at high baryon to meson ratio (energies of the SPS and below), the antihyperon yield is small and the main carriers of antistrange quarks are $\mathrm{K}^{+}$and $\mathrm{K}^{0}$ with $\left\langle\mathrm{K}^{+}\right\rangle \approx\left\langle\mathrm{K}^{0}\right\rangle$ due to approximate isospin symmetry in heavy-ion collisions. Thus, the $\mathrm{K}^{+}$yield counts about half of the strange quark-antiquark pairs $(\langle s \bar{s}\rangle)$ produced in the collisions and contained in the reaction products [7]. In contrast, fractions of strange quarks carried by $\mathrm{K}^{-}, \overline{\mathrm{K}}^{0}$, and hyperons are comparable and change significantly with the baryon to meson ratio. At lower collision energies, relatively more strange quarks are carried by hyperons and less by antikaons. Thus, the energy dependence of the $\mathrm{K}^{-}$yield does not follow the energy dependence of $\langle s \bar{s}\rangle$. This is illustrated
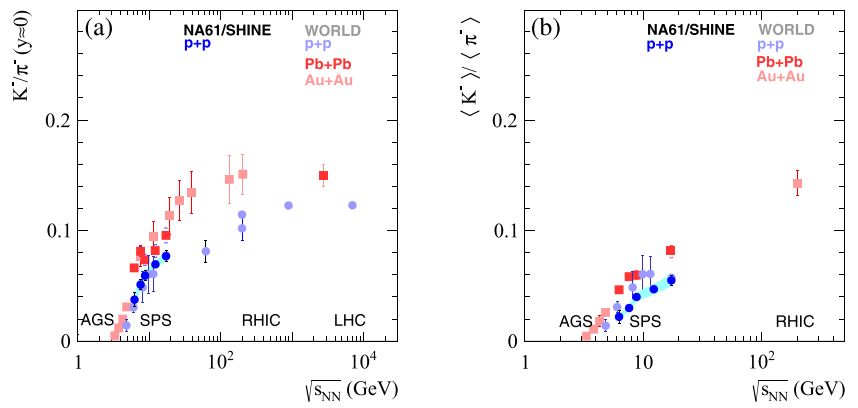

FIG. 2. Energy dependence of (a) the $\mathrm{K}^{-} / \pi^{-}$ratio at midrapidity and (b) in the full phase space. The results for the inelastic $p+p$ interactions of the NA61/SHINE Collaboration (blue circles) are shown together with the world data on $p+p$ interactions (light blue circles) as well as central $\mathrm{Pb}+\mathrm{Pb}$ (red squares) and $\mathrm{Au}+\mathrm{Au}$ (light red squares) collisions [21,23,25-38]. The points of the NA61/SHINE Collaboration include statistical uncertainties, and the systematic uncertainties are marked as a shaded band. For the $\mathrm{K}^{-} / \pi^{-}$and $\left\langle\mathrm{K}^{-}\right\rangle /\left\langle\pi^{-}\right\rangle$ratio, the total uncertainties are marked for the points of the $(\mathrm{Pb}+\mathrm{Pb})$ interactions of the NA49 Collaboration, the $(\mathrm{Au}+\mathrm{Au})$ interactions of the STAR Collaboration, the $(p+p)$ interactions of the PHENIX Collaboration, the $(p+p$ and $\mathrm{Pb}+\mathrm{Pb})$ interactions of the ALICE Collaboration, and the $(\mathrm{Au}+\mathrm{Au})$ experiments of the BRAHMS Collaboration whereas only statistical uncertainties are plotted for the remaining points.

in Fig. 2 where the energy dependence of the $\mathrm{K}^{-} / \pi^{-}$ratio at midrapidity (a) and in the full phase space (b) for inelastic $p+p$ interactions and heavy-ion collisions is shown. In conclusion, the $\mathrm{K}^{+}$yield is preferred over $\mathrm{K}^{-}$and $\Lambda$ yields when the total number of $s \bar{s}$ pairs is of interest as in the search for the QGP [40] and the onset of deconfinement [8].

The collision energy dependence of the $\mathrm{K}^{+} / \pi^{+}$ratio in inelastic $p+p$ interactions is different from the one in heavyion collisions, see Fig. 1. First of all, the ratio is smaller in $p+p$ interactions than in $\mathrm{Pb}+\mathrm{Pb}$ and $\mathrm{Au}+\mathrm{Au}$ collisions and does not show the horn structure.

The $p+p$ ratio approaches that in heavy-ion reactions with increasing energy, at the LHC, it is only about $20 \%$ smaller than the corresponding ratio for central $\mathrm{Pb}+\mathrm{Pb}$ collisions. Starting from the threshold energy, the ratio in $p+p$ interactions steeply increases to reach a plateau at energies of the CERN SPS. The plateau is followed by a weak increase towards energies of the LHC. Notably, the beginning of the plateau in $p+p$ interactions coincides with the horn maximum in heavy-ion collisions.

According to the standard model of heavy-ion collisions, the inverse slope parameter $T$ obtained from exponential fits of transverse mass spectra is sensitive to both the temperature and the radial flow in the final state. The energy dependence of $T$ of the transverse mass spectra of $\mathrm{K}^{+}$and $\mathrm{K}^{-}$mesons produced at midrapidity in inelastic $p+p$ interactions is presented in Figs. 1(c) and 1(d), respectively. The results of the NA61/SHINE Collaboration [17] are compared to the world data for $p+p$ and heavy-ion collisions [21,23,25-38]. Unless the $T$ parameter was given directly by the experiment, it was taken from Ref. [21] or determined from transverse mass/momentum spectra according to the procedure of 

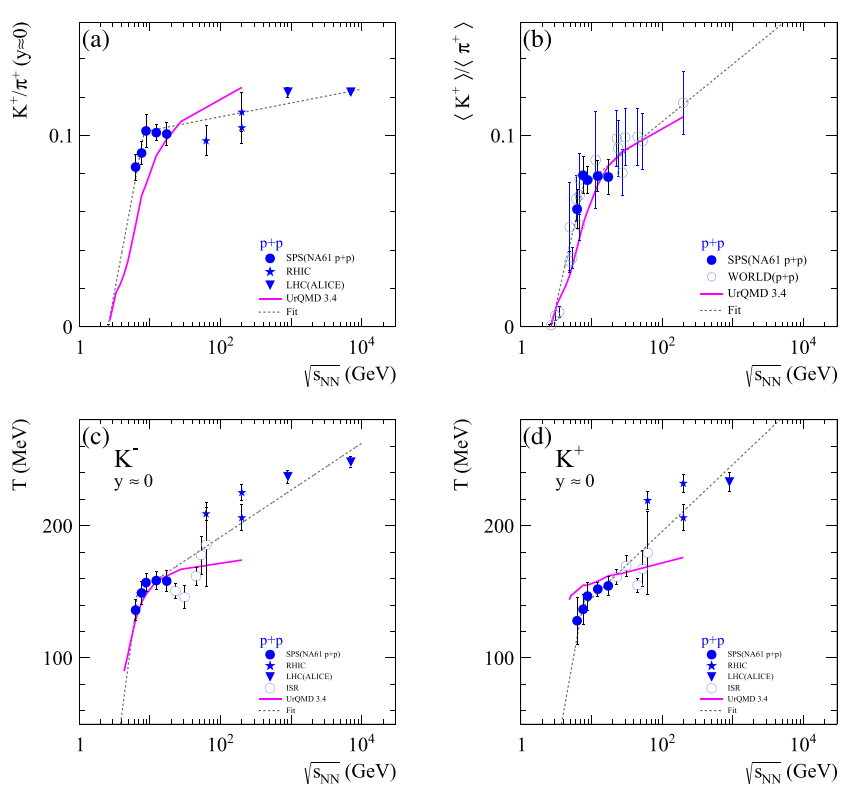

FIG. 3. Energy dependence of (a) the $\mathrm{K}^{+} / \pi^{+}$ratio in inelastic $p+p$ interactions at midrapidity and (b) in the full phase space as well the inverse slope parameter $T$ of transverse mass spectra at midrapidity for (c) $\mathrm{K}^{-}$and (d) $\mathrm{K}^{+}$mesons. The data are fitted by two straight lines in order to locate the position of the break in the energy dependence. The experimental results are compared with predictions of the resonance-string model Ultrarelativistic Quantum Molecular Dynamics (UrQMD) [42]. Only statistical uncertainties are plotted and used for the fit.

Ref. [21]. The collision energy dependence of the $T$ parameter in heavy-ion collisions shows the so-called step structure. Following a fast rise, the $T$ parameter passes through a stationary region, which starts at the low energies of the SPS and then (above the top energy of the SPS) enters a domain of a steady increase. The increase continues up to the top energy of the LHC. The step was predicted as a signal of the onset of deconfinement $[8,41]$ resulting from the softening of the equation of state in the transition region. The collision energy dependence of the $T$ parameter in inelastic $p+p$ interactions is similar to the one for central $\mathrm{Pb}+\mathrm{Pb}$ and $\mathrm{Au}+\mathrm{Au}$ collisions. The main difference is that the $T$ parameter in $p+p$ interactions is significantly smaller than for heavy-ion collisions which is usually attributed to smaller radial flow.

To estimate the break energy between a fast rise at low energies and a plateau or slower increase at high energies two straight lines were fitted to the $p+p$ data (see Fig. 3). The low-energy line was constrained by the threshold energy for kaon production. The fitted break energy is $8.3 \pm 0.6,7.70 \pm 0.14,6.5 \pm 0.5$, and $7.9 \pm 0.2 \mathrm{GeV}$ for the $\mathrm{K}^{+} / \pi^{+},\left\langle\mathrm{K}^{+}\right\rangle /\left\langle\pi^{+}\right\rangle$ratios and $T\left(\mathrm{~K}^{-}\right), T\left(\mathrm{~K}^{+}\right)$, respectively. These values are close to each other and surprisingly close to the energy of the beginning of the horn and step structures in central $\mathrm{Pb}+\mathrm{Pb}$ collisions-the transition energy being approximately $8 \mathrm{GeV}$ (see Fig. 1).

Figure 3 presents also predictions of a resonance-string model UrQMD [42]. This model assumes the transition between particle production by resonance formation at low energies and string formation at high energies [43]-the resonance-string transition. The postulate allows to approximately fit the fast low-energy increase in the $\mathrm{K}^{+} / \pi^{+}$ratio and the inverse slope parameter of transverse mass spectra of charged kaons and its slowing down at high energies. The sharpness of the break is not reproduced by the model.

The unexpected similarity of the transition energy in central $\mathrm{Pb}+\mathrm{Pb}$ collisions and the break energy in $p+p$ interactions provoke the question whether there is a common physics origin of the two effects. Is this coincidence accidental? If not, do we see effects included in standard modeling of heavy-ion collisions in $p+p$ interactions, or reversely nonequilibrium processes in $p+p$ interactions lead to the horn and step in heavy-ion collisions? The interpretation of these results is still under vivid discussion. An obstacle is that the validity of quantitative models is usually restricted to a limited range in collision energy, size of colliding nuclei and concerns only selected observables. Examples of recent developments are given in Refs. [44-49].

To summarize, new results of the NA61/SHINE Collaboration on the collision energy dependence of the $\mathrm{K}^{+} / \pi^{+}$ ratio and the inverse slope parameter of kaon $m_{\mathrm{T}}$ spectra in inelastic $p+p$ interactions are presented together with a compilation of the world data. The $p+p$ results are compared with the corresponding measurements in central $\mathrm{Pb}+\mathrm{Pb}$ and $\mathrm{Au}+\mathrm{Au}$ collisions. The comparison uncovers a similarity between the collision energy dependence in $p+p$ interactions and central heavy-ion collisions - a rapid change in collision energy dependence of basic hadron production properties in the same energy range. At the same time, they confirm with higher significance previously reported striking differences between results for $p+p$ interactions and heavy-ion collisions. Possible interpretations are briefly discussed. Clearly, understanding of the origin for the similarity between results on heavy-ion collisions and $p+p$ interactions is one of the key objectives of heavy-ion physics today. Emerging results from the NA61/SHINE Collaboration (nuclear mass number)-(collision energy) scan as well as results from the LHC and the Relativistic Heavy-Ion Collider (RHIC) on collisions of small- and medium-size systems qualitatively change the experimental landscape. In parallel, significant progress is needed in the modeling of collision energy and system-size dependence which would extend the validity of models to the full range covered by the data.

We would like to thank the CERN EP, BE, HSE, and EN Departments for the strong support of the NA61/SHINE Collaboration. This work was supported by the Hungarian Scientific Research Fund (Grant No. NKFIH 123842/123959), the Polish Ministry of Science and Higher Education the National Science Centre Poland (Grants No. 2011/03/N/ST2/03691, No. 2013/11/N/ ST2/03879, No. 2014/13/N/ST2/02565, No. 2014/14/E/ ST2/00018, No. 2014/15/B/ST2/02537, No. 2015/18/M/ ST2/00125, No. 2015/19/N/ST2/01689, No. 2016/23/B/ ST2/00692, No. 2017/25/N/ST2/02575, No. 2018/30/A/ST2/ 00226, and No. 2018/31/G/ST2/03910), the Russian Science Foundation, Grant No. 16-12-10176, the Russian Academy 
of Science and the Russian Foundation for Basic Research (Grants No. 08-02-00018, No. 09-02-00664, and No. 1202-91503-CERN), the Ministry of Science and Education of the Russian Federation, Grant No. 3.3380.2017/4.6, the National Research Nuclear University MEPhI in the framework of the Russian Academic Excellence Project (Contracts No. 02.a03.21.0005 and No. 27.08.2013), the Ministry of Education, Culture, Sports, Science and Technology, Japan, Grant-in-Aid for Scientific Research (Grants No. 18071005, No. 19034011, 19740162, No. 20740160, and No. 20039012), the German Research Foundation (Grant No. GA 1480/8-
1), the Bulgarian Nuclear Regulatory Agency and the Joint Institute for Nuclear Research, Dubna (bilateral Contract No. 4799-1-18/20), Bulgarian National Science Fund (Grant No. DN08/11), Ministry of Education and Science of the Republic of Serbia (Grant No. OI171002), Swiss Nationalfonds Foundation (Grant No. 200020117913/1), ETH Research Grant No. TH-01 07-3, and the Fermi National Accelerator Laboratory (Fermilab), a U.S. Department of Energy, Office of Science, HEP User Facility. Fermilab is managed by Fermi Research Alliance, LLC (FRA), acting under Contract No. DE-AC02-07CH11359.
[1] W. Florkowski, Phenomenology of Ultra-Relativistic Heavy-Ion Collisions (World Scientific, Singapore, 2010).

[2] G. Torrieri, S. Steinke, W. Broniowski, W. Florkowski, J. Letessier, and J. Rafelski, Comput. Phys. Commun. 167, 229 (2005).

[3] F. Becattini, J. Manninen, and M. Gazdzicki, Phys. Rev. C 73, 044905 (2006).

[4] A. Andronic, P. Braun-Munzinger, K. Redlich, and J. Stachel, Nature (London) 561, 321 (2018).

[5] S. Afanasiev et al. (NA49 Collaboration), Phys. Rev. C 66 , 054902 (2002).

[6] C. Alt et al. (NA49 Collaboration), Phys. Rev. C 77, 024903 (2008).

[7] M. Gazdzicki, M. Gorenstein, and P. Seyboth, Acta Phys. Pol., B 42, 307 (2011).

[8] M. Gazdzicki and M. I. Gorenstein, Acta Phys. Pol., B 30, 2705 (1999).

[9] B. Andersson, G. Gustafson, G. Ingelman, and T. Sjostrand, Phys. Rep. 97, 31 (1983).

[10] V. V. Begun, M. Gazdzicki, and M. I. Gorenstein, Phys. Rev. C 78, 024904 (2008).

[11] S. Chatrchyan et al. (CMS Collaboration), Phys. Lett. B 718 , 795 (2013).

[12] V. Khachatryan et al. (CMS Collaboration), Phys. Lett. B 765, 193 (2017).

[13] J. L. Nagle and W. A. Zajc, Annu. Rev. Nucl. Part. Sci. 68, 211 (2018).

[14] P. Bozek and W. Broniowski, PoS LHCP2016, 116 (2016).

[15] J. Adam et al. (ALICE Collaboration), Nat. Phys. 13, 535 (2017).

[16] N. Abgrall et al. (NA61/SHINE Collaboration), J. Instrum. 9, P06005 (2014).

[17] A. Aduszkiewicz et al. (NA61/SHINE Collaboration), Eur. Phys. J. C 77, 671 (2017).

[18] A. Aduszkiewicz et al. (NA61/SHINE Collaboration), Eur. Phys. J. C 76, 635 (2016).

[19] M. Gazdzicki and D. Roehrich, Z. Phys. C 65, 215 (1995).

[20] M. Gazdzicki and D. Rohrich, Z. Phys. C 71, 55 (1996).

[21] M. Kliemant, B. Lungwitz, and M. Gazdzicki, Phys. Rev. C 69 , 044903 (2004).

[22] I. Arsene et al. (BRAHMS Collaboration), Phys. Rev. C 72, 014908 (2005).

[23] K. Aamodt et al. (ALICE Collaboration), Eur. Phys. J. C 71 1655 (2011).

[24] B. Abelev et al. (ALICE Collaboration), Phys. Rev. Lett. 109, 252301 (2012).
[25] B. Alper et al. (British-Scandinavian), Nucl. Phys. B 100, 237 (1975).

[26] B. Abelev et al. (STAR Collaboration), Phys. Rev. C 79, 034909 (2009).

[27] B. B. Abelev et al. (ALICE Collaboration), Phys. Lett. B 736, 196 (2014).

[28] L. Ahle et al. (E802 Collaboration), Phys. Rev. C 57, R466(R) (1998).

[29] L. Ahle et al. (E-802 Collaboration), Phys. Rev. C 58, 3523 (1998).

[30] L. Ahle et al. (E-802 and E-866 Collaborations), Phys. Rev. C 60, 044904 (1999).

[31] L. Ahle et al. (E866 and E917 Collaborations), Phys. Lett. B 476, 1 (2000).

[32] L. Ahle et al. (E866 and E917 Collaborations), Phys. Lett. B 490, 53 (2000).

[33] J. Barrette et al. (E877 Collaboration), Phys. Rev. C 62, 024901 (2000).

[34] D. Pelte et al. (FOPI Collaboration), Z. Phys. A 357, 215 (1997).

[35] I. Bearden et al. (BRAHMS Collaboration), Phys. Rev. Lett. 94, 162301 (2005).

[36] L. Adamczyk et al. (STAR Collaboration), Phys. Rev. C 96, 044904 (2017).

[37] B. Abelev et al. (ALICE Collaboration), Phys. Rev. C 88, 044910 (2013).

[38] J. Adam et al. (ALICE Collaboration), Eur. Phys. J. C 75, 226 (2015).

[39] A. Adare et al. (PHENIX Collaboration), Phys. Rev. C 83, 064903 (2011).

[40] P. Koch, B. Muller, and J. Rafelski, Phys. Rep. 142, 167 (1986).

[41] M. I. Gorenstein, M. Gazdzicki, and K. A. Bugaev, Phys. Lett. B 567, 175 (2003).

[42] M. Bleicher et al., J. Phys. G: Nucl. Part. Phys. 25, 1859 (1999).

[43] V. Yu. Vovchenko, D. V. Anchishkin, and M. I. Gorenstein, Nucl. Phys. A 936, 1 (2015).

[44] W. Cassing, A. Palmese, P. Moreau, and E. L. Bratkovskaya, Phys. Rev. C 93, 014902 (2016).

[45] P. Batyuk, D. Blaschke, M. Bleicher, Yu. B. Ivanov, I. Karpenko, S. Merts, M. Nahrgang, H. Petersen, and O. Rogachevsky, Phys. Rev. C 94, 044917 (2016).

[46] J. Weil et al., Phys. Rev. C 94, 054905 (2016).

[47] J. Jalilian-Marian, Nucl. Phys. A 982, 935 (2019).

[48] R. D. Weller and P. Romatschke, Phys. Lett. B 774, 351 (2017).

[49] A. Kurkela, U. A. Wiedemann, and B. Wu, Eur. Phys. J. C 79, 965 (2019) 